\title{
Genome-wide identification and characterization of the NF- $Y$ gene family in grape (vitis vinifera L.)
}

\author{
Chong Ren ${ }^{1,2}$, Zhan Zhang ${ }^{1,2}$, Yi Wang ${ }^{1,2}$, Shaohua Li ${ }^{1 *}$ and Zhenchang Liang ${ }^{1 *}$
}

\begin{abstract}
Background: Nuclear factor $Y(N F-Y)$ transcription factor is composed of three distinct subunits: NF-YA, NF-YB and NF-YC. Many members of NF-Y family have been reported to be key regulators in plant development, phytohormone signaling and drought tolerance. However, the function of the NF-Y family is less known in grape (Vitis vinifera L.).

Results: A total of 34 grape NF-Y genes that distributed unevenly on grape ( $V$. vinifera) chromosomes were identified in this study. Phylogenetic analysis was performed to predict functional similarities between Arabidopsis thaliana and grape NF-Y genes. Comparison of the structures of grape NF-Y genes (VVNF-Ys) revealed their functional conservation and alteration. Furthermore, we investigated the expression profiles of VVNF-YS in response to various stresses, phytohormone treatments, and in leaves and grape berries with various sugar contents at different developmental stages. The relationship between VVNF-Y transcript levels and sugar content was examined to select candidates for exogenous sugar treatments. Quantitative real-time PCR ( $P P C R$ ) indicated that many VvNF-Ys responded to different sugar stimuli with variations in transcript abundance. QPCR and publicly available microarray data suggest that VVNF-YS exhibit distinct expression patterns in different grape organs and developmental stages, and a number of VVNF-YS may participate in responses to multiple abiotic and biotic stresses, phytohormone treatments and sugar accumulation or metabolism.
\end{abstract}

Conclusions: In this study, we characterized 34 VVNF-Ys based on their distributions on chromosomes, gene structures, phylogenetic relationship with Arabidopsis NF-Y genes, and their expression patterns. The potential roles of VVNF-YS in sugar accumulation or metabolism were also investigated. Altogether, the data provide significant insights on VVNF-YS, and lay foundations for further functional studies of NF-Y genes in grape.

Keywords: Grape (Vitis vinifera L.), NF-Y transcription factor, Phylogenetic analysis, Expression profiles, Quantitative real-time PCR

\section{Background}

NF-Y (for Nuclear factor Y) transcription factors (TFs) are almost found in all eukaryotes, and they are involved in regulation of gene expression by binding the CCAAT element $[1,2]$. The NF-Y complex known as CCAAT binding factor $(\mathrm{CBF})$ or heme activator protein (HAP) consists of three distinct subunits: NF-YA (also known as CBF-B or HAP2), NF-YB (CBF-A or HAP3) and NF-

\footnotetext{
* Correspondence: shhli@ibcas.ac.cn; ZL249@ibcas.ac.cn

${ }^{1}$ Beijing Key Laboratory of Grape Science and Enology and Key Laboratory of Plant Resource, Institute of Botany, the Chinese Academy of Sciences, Beijing 100093, People's Republic of China

Full list of author information is available at the end of the article
}

YC (CBF-C or HAP5) [3]. All subunits contain evolutionarily-conserved DNA binding and subunit interaction domains to form heterotrimeric complexes [4-6]. Notably, the NF-YB proteins without a nuclear localization signal (NLS) have to interact with NF-YC in the cytoplasm to translocate into the nucleus, where the heterodimer is combined with NF-YA to form the final heterotrimer $[7,8]$. Despite the ubiquity of NF-Y proteins in eukaryotes, there is only one or two genes encoding each NF-Y subunit in animals and yeast $[9,10]$. In contrast, there are multiple genes encoding each subunit in plants $[10,11]$. For example, 10 NF-YAs, 13 NF-YBs, and 13 NF-YCs are encoded by the Arabidopsis thaliana 
genome $[1,10]$. This expansion is a common feature in the plant kingdom, and it helps plants form flexible, versatile TF systems to accommodate complex and diverse environment conditions [11].

As a kind of combinatorial TFs, NF-Ys have been reported to be involved in regulation of plant development and respond to various abiotic and biotic stresses [12-19]. The Arabidopsis LEAFY COTYLEDON 1 (LEC1, AtNF$Y B 9)$ is the first cloned and well-known plant $N F-Y$ gene, and it has been proven that LEC1 is a pivotal regulator in embryogenesis [12, 13, 20, 21]. Recently, the NF-Y genes are also found to be involved in response to endoplasmic reticulum (ER) stress [22, 23]. Grape (Vitis spp.) is cultivated worldwide and has tremendous economic value, and a few reports have emerged revealing the role of $V v L 1 L$ in grape [24, 25]. However, the function of the overwhelming majority of $N F-Y$ genes in grape is still poorly understood, despite the conservation of functional amino acid residues across different species [26-28].

To explore and characterize the potential functions of grape $N F-Y$ genes $(V v N F-Y s)$, we adopted bioinformatics to analyze the 34 identified $V v N F-Y s$ (8 NF-YAs, 18 $N F-Y B s, 8 N F-Y C s$ ) based on publicly available data. Furthermore, we investigated the expression patterns of $V v N F-Y s$ in response to different biotic and abiotic stresses, exogenous phytohormone, and sugar treatments. In addition, the expression profiles of $V v N F-Y_{S}$ in grape berries were examined at different developmental stages. The phylogenetic analysis of NF-YS from grape and Arabidopsis, investigation of protein motif and exon-intron structure patterns, and the experimental data provide insights on the function of $V v N F-Y_{s}$. Taken together, our results provide a set of candidate $N F-Y$ genes for future study and genetic modification in grape.

\section{Results}

Identification and characterization of grape $N F-Y$ genes

NF-Y proteins were identified by searching the Plant Transcription Factor Database (PlantTFDB, http:// planttfdb.cbi.pku.edu.cn/) and the UniProt database (http://www.uniprot.org/) using the PFAM and KOG IDs of conserved domains. Then, a BLAST search of the $12 \times$ grape genome was performed using fulllength amino acid sequences of candidate $N F-Y$ genes. By removing incomplete and redundant sequences, 34 $N F-Y$ genes were identified, including $8 N F-Y A, 18$ $N F-Y B$, and $8 N F-Y C$ genes (Table 1$)$. The $34 V v N F-$ $Y S$ were named based on their distribution and relative distance on grape chromosomes. Thirty-two $V v N F-Y s$ could be mapped on 14 grape chromosomes with the exception of $V v N F-Y B 17$ and $V v N F-Y B 18$ (Table 1 and Additional file 1: Figure S1). Among these chromosomes, four possessed only one $N F-Y$ gene, and seven possessed two NF- $Y$ genes. Chromosomes 6 and 19 had five $N F-Y$ genes, most of which were concentrated in the upper part of the chromosomes (Additional file 1: Figure S1). Uneven and variable distribution of $V v N F-Y s$ on grape chromosomes is consistent with the results of previous reports [3, 29].

Characteristics of the $34 V v N F-Y s$ are shown in Table 1. Significant difference of the length of $V v N F-Y$ sequences was observed, with a range from 351 to $51,220 \mathrm{bp}$, and the difference results in variability of predicted amino acid numbers. The exon-intron structures of $V v N F-Y s$ were also analyzed (Additional file2: Figure S2). The exon-intron organization could indicate the evolutionary relationships within multi-gene families [30]. Most of $V v N F-Y A s$ had five or six exons and four or five introns and their intron phases occurred in the same pattern except for $V v N F-Y A 1$ and $V v N F-Y A 8$. The structures of $V v N F-Y B s$ and $V v N F-Y C s$ were more variable and complicated, and the two families shared similar exon-intron organization (Additional file 2: Figure S2). The results were consistent with the previous report [29].

\section{Phylogenetic analysis and multiple alignment of NF-Y protein sequences}

To investigate the evolutionary relationship and functional association of $V v N F-Y S$ with Arabidopsis NF-Y family, we constructed an unrooted phylogenetic tree using the protein sequences of NF-Ys from grape and Arabidopsis (Fig. 1). The phylogenetic analysis showed that the $34 V v N F-Y S$ were divided into three groups (Fig. 1). Almost all the members of $V v N F-Y A s, V v N F-Y B s$ or $V v N F-Y C s$ were clustered into the same sub-branch except for $V v N F-Y C 3$. Three pairs of $N F-Y A s$, four pairs of $N F-Y B S$ and one pair of $N F-Y C s$ showed high similarity in sequence, respectively (Fig. 1). Most of $V v N F-Y s$ had homologs in Arabidopsis. The phylogenetic relationship indicated basal architecture conservation and possible functional similarities of NF-Y family between grape and Arabidopsis.

Multiple sequence alignments of NF-Y proteins from grape, Arabidopsis, human (Homo sapiens), mouse (Rattus norvegicus) and yeast (Saccharomyces cerevisiae) were generated. NF-YA proteins which lack distinct homology to other annotated proteins [30] were characterized by two conserved domains: the DNA-binding domain and the subunit interaction domain [31, 32]. The two domains were conserved among plants and other eukaryote organisms (Fig. 2). The amino acid residues required for functionality in most mammals and yeast $[31,32]$ were present in grape NF-YA proteins (Fig. 2a). The conservation of functionality required amino acid residues across different eukaryote lineages strongly suggests functional conservation [1]. As with NF-YA proteins, NF-YBs and NF-YCs 
Table 1 NF-Y transcription factors in grape

\begin{tabular}{|c|c|c|c|c|c|c|c|}
\hline Name & Gene ID & Best match in Arabidopsis & Chr. & Strand & Genomic (bp) & No. of aa & $\mathrm{pl}$ \\
\hline \multicolumn{8}{|c|}{ NF-YA Subunit } \\
\hline NF-YA1 & GSVIVT01036936001 & At1g31420 & 2 & + & 51220 & 1611 & 8.89 \\
\hline NF-YA2 & GSVIVT01025252001 & At5g12840, AtNF-YA1 & 6 & + & 7100 & 306 & 6.49 \\
\hline NF-YA3 & GSVIVT01033313001 & At3g20910, AtNF-YA9 & 8 & + & 5639 & 354 & 8.67 \\
\hline NF-YA4 & GSVIVT01022601001 & At5g06510, AtNF-YA10 & 8 & - & 8762 & 309 & 9.34 \\
\hline NF-YA5 & GSVIVT01016790001 & At1g72830, AtNF-YA3 & 9 & + & 7541 & 336 & 9.15 \\
\hline NF-YA6 & GSVIVT01021622001 & At1g30500, AtNF-YA7 & 10 & + & 14679 & 208 & 7.17 \\
\hline NF-YA7 & GSVIVT01015120001 & At3g14020, AtNF-YA6 & 11 & + & 2972 & 310 & 8.76 \\
\hline NF-YA8 & GSVIVT01032101001 & At3g20910, AtNF-YA9 & 13 & - & 8954 & 405 & 9.30 \\
\hline \multicolumn{8}{|c|}{ NF-YB Subunit } \\
\hline NF-YB1 & GSVIVT01010260001 & At1g09030, AtNF-YB4 & 1 & + & 459 & 152 & 5.78 \\
\hline NF-YB2 & GSVIVT01010264001 & At1g09030, AtNF-YB4 & 1 & + & 459 & 152 & 8.87 \\
\hline NF-YB3 & GSVIVT01017741001 & At2g47810, AtNF-YB5 & 5 & - & 417 & 138 & 5.16 \\
\hline NF-YB4 & GSVIVT01036120001 & At2g27470, AtNF-YB11 & 6 & + & 8280 & 193 & 5.59 \\
\hline NF-YB5 & GSVIVT01025110001 & At3g53340, AtNF-NB10 & 6 & - & 2009 & 133 & 6.83 \\
\hline NF-YB6 & GSVIVT01022214001 & At5g64950 & 7 & + & 876 & 291 & 9.07 \\
\hline NF-YB7 & GSVIVT01010959001 & At2g47810, AtNF-YB5 & 7 & + & 387 & 128 & 6.84 \\
\hline NF-YB8 & GSVIVT01025539001 & At2g37060, AtNF-YB8 & 8 & + & 2436 & 161 & 5.81 \\
\hline NF-YB9 & GSVIVT01030085001 & At4g12730 & 12 & + & 7125 & 482 & 5.93 \\
\hline NF-YB10 & GSVIVT01016347001 & At2g37060, AtNF-YB8 & 13 & + & 4682 & 176 & 6.42 \\
\hline NF-YB11 & GSVIVT01031089001 & At1g09030, AtNF-YB4 & 14 & + & 444 & 147 & 6.83 \\
\hline NF-YB12 & GSVIVT01008215001 & At5g23090, AtNF-YB13 & 17 & - & 8526 & 155 & 4.62 \\
\hline NF-YB13 & GSVIVT01014672001 & At5g55660 & 19 & + & 11644 & 1098 & 6.36 \\
\hline NF-YB14 & GSVIVT01014673001 & At5g47640, AtNF-YB2 & 19 & + & 1711 & 210 & 6.44 \\
\hline NF-YB15 & GSVIVT01014689001 & At5g47670, AtNF-YB6 & 19 & + & 1402 & 209 & 5.89 \\
\hline NF-YB16 & GSVIVT01014690001 & At5g47670, AtNF-YB6 & 19 & + & 1537 & 215 & 5.48 \\
\hline NF-YB17 & GSVIVT01004375001 & At4g14540, AtNF-YB3 & Un & + & 1376 & 114 & 4.10 \\
\hline NF-YB18 & GSVIVT01002895001 & At5g47670, AtNF-YB6 & Un & - & 2458 & 211 & 5.91 \\
\hline \multicolumn{8}{|c|}{ NF-YC Subunit } \\
\hline NF-YC1 & GSVIVT01019784001 & At1g54830, AtNF-YC3 & 2 & + & 351 & 116 & 9.46 \\
\hline NF-YC2 & GSVIVT01017901001 & At1g08970, AtNF-YC9 & 5 & + & 4358 & 104 & 9.10 \\
\hline NF-YC3 & GSVIVT01025169001 & At1g07980, AtNF-YC10 & 6 & + & 8437 & 425 & 9.72 \\
\hline NF-YC4 & GSVIVT01037394001 & At3g12480, AtNF-YC11 & 6 & - & 8197 & 301 & 4.92 \\
\hline NF-YC5 & GSVIVT01036581001 & At3g12480, AtNF-YC11 & 13 & + & 15144 & 271 & 9.40 \\
\hline NF-YC6 & GSVIVT01030963001 & At5g63470, AtNF-YC4 & 14 & - & 946 & 129 & 6.36 \\
\hline NF-YC7 & GSVIVT01008570001 & At3g48590, AtNF-YC1 & 17 & - & 4379 & 215 & 4.95 \\
\hline NF-YC8 & GSVIVT01036760001 & At1g56170, AtNF-YC2 & 19 & - & 1072 & 114 & 5.06 \\
\hline
\end{tabular}

contained DNA-binding and subunit interaction domains as well (Fig. 2b, c). The required amino acids were wellconserved in most of grape NF-YB proteins except for NF-YB7 and NF-YB17. The aspartate $\left(D_{72}\right)$ which is thought to be significant for protein interactions $[28,33]$ was conserved in almost all NF-YB proteins (Fig. 2b). Half of grape NF-YC proteins exhibited residue deletions, and some residues in NF-YC were replaced by alternative ones of similar properties. However, the arginine $\left(R_{52}\right)$ and aspartate $\left(D_{59}\right)$ which are necessary for stabilization of NF-YB/C [28] were present in most NF-YCs (Fig. 2c). The conservation of protein sequences suggests the conserved function while the non-conservative changes would indicate novel functional alterations [1]. 


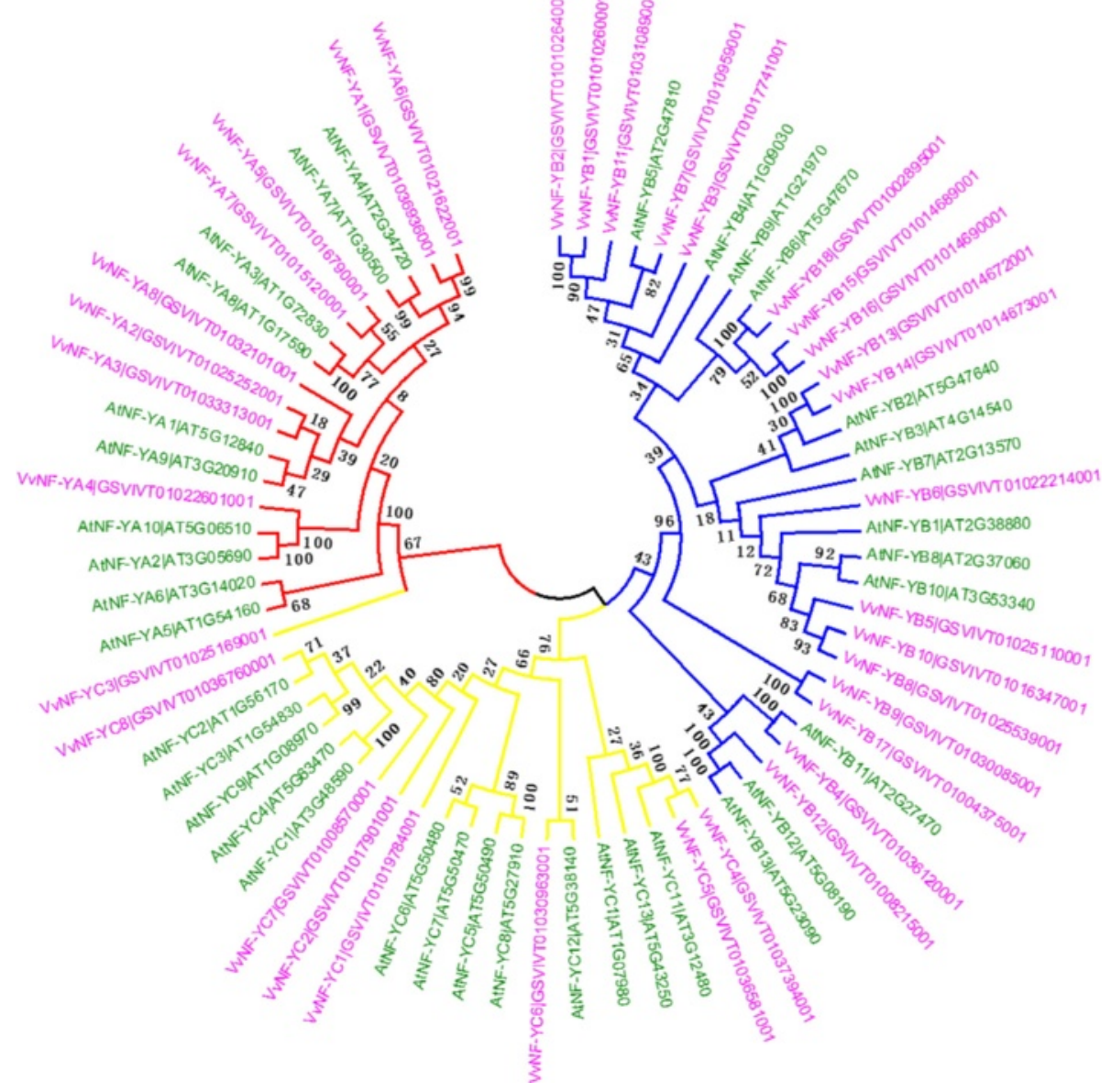

Fig. 1 Phylogenetic analysis of NF-Y proteins from grape and Arabidopsis thaliana. Thirty-four NF-Y proteins from grape and 36 NF-Y proteins from Arabidopsis were divided into four branches according to subunit type. Red branch indicates NF-YAs, blue branch represents NF-YBs, and two yellow branches denote NF-YCS

\section{Expression patterns of VvNF-Ys in response to abiotic,} biotic stresses and phytohormones

Numerous reports have revealed the function of individual $N F-Y$ genes in responses to various biotic and abiotic stresses [14, 18, 19, 34, 35]. To further investigate how $N F-Y$ genes response to stresses, expression of $V v N F-Y$ genes was measured under several stresses. Probe sets from Affymetrix GeneChip platform for $16 V v N F-Y_{S}$ (Additional file 3: Table S1) were successfully obtained and corresponding genes were selected for further study.

We first examined the responsiveness of $V \nu N F-Y_{S}$ to multiple abiotic stresses including salt, drought, cold and high temperature by taking advantage of publicly available data. 'Cabernet Sauvignon' (Vitis vinifera L.) plants were treated with salt, drought (PEG) and cold $\left(5{ }^{\circ} \mathrm{C}\right)$, respectively, and expression of $V v N F-Y_{S}$ was analyzed subsequently. About three $V v N F-Y_{S}$ (1 $V v N F-Y A$ and $2 V v N F-Y B s)$ were up- or down-regulated ( $\geq 1$-fold) to at least one stress treatment (Fig. 3). $V v N F-Y A 3$ was induced and the transcript level reached a peak of nearly 2-fold at $24 \mathrm{~h}$ after salt and PEG treatments, while $V v N F-Y B 18$ responded to all the stress treatments and its expression was rapidly suppressed ( $>2$-fold) at $4 \mathrm{~h}$ after the treatments (Fig. 3a). The difference in expression patterns of $V v N F-Y A 3$ and $V v N F-Y B 18$ suggests their different roles in response to salt and PEG. For heat treatment, 'Cabernet Sauvignon' seedlings derived from stem cuttings were placed at $45{ }^{\circ} \mathrm{C}$ and then recovered at $25{ }^{\circ} \mathrm{C}$ [36]. Two $V v N F-Y B s$ responded ( $\geq 2$-fold) to heat stress or the following recovery process (Fig. $3 \mathrm{~b}$ ). The transcript abundance of $V v N F-Y B 18$ was increased ( $\geq 2$-fold) in response to heat treatment, whereas the other gene, $V v N F-Y B 9$, was down-regulated ( $\geq 1$-fold) after the heat treatment. However, the transcript level of $V v N F-Y B 9$ was increased ( $\geq 2$-fold) again during the subsequent recovery process (Fig. $3 \mathrm{~b}$ ). These results showed that $V v N F-Y B 18$ may help enhance the resistance of grape to heat stress, and $V v N F-Y B 9$ may participate in the heat recovery process. All the results discussed above revealed that some of $V v N F-Y s$ may be associated with 


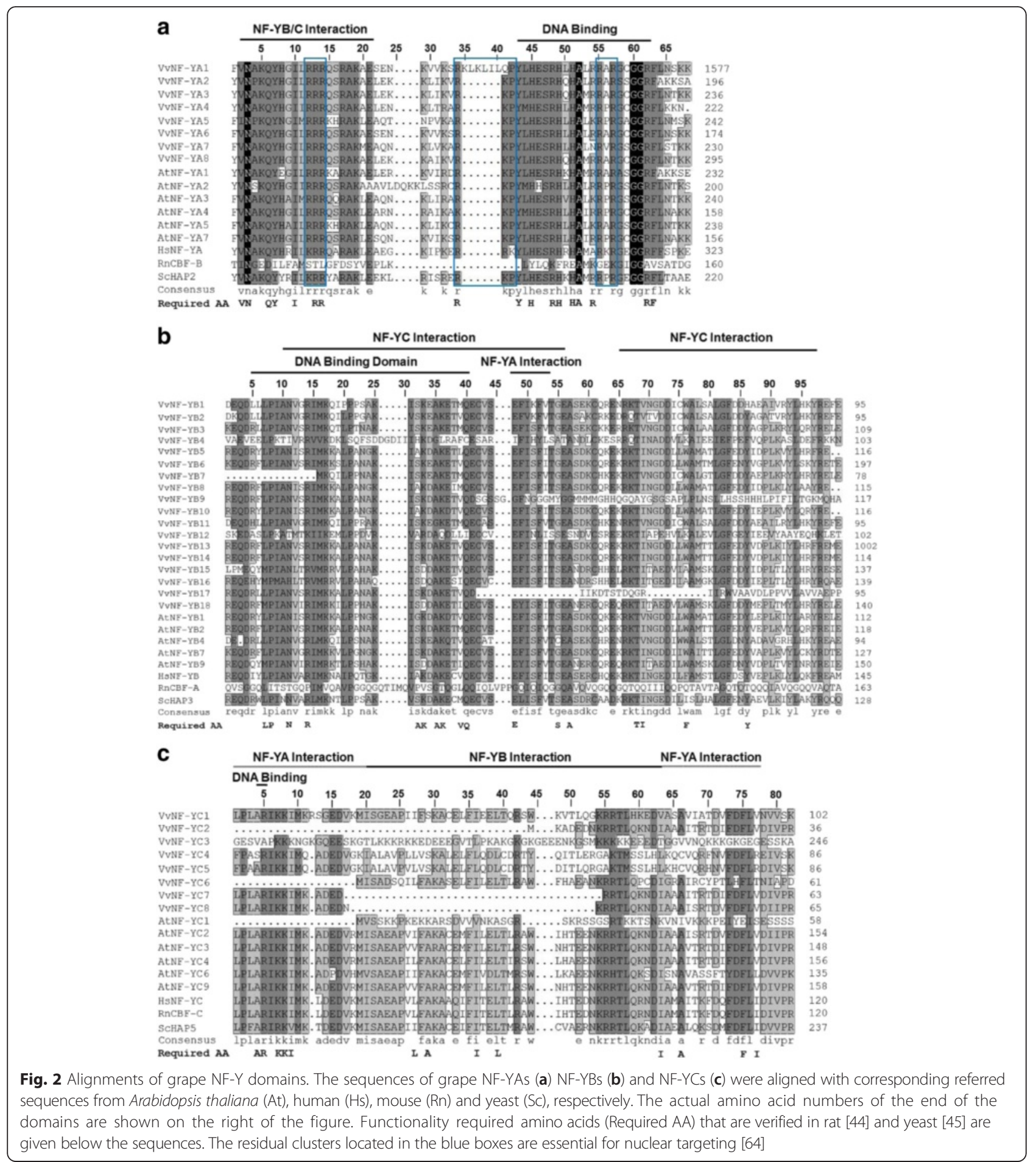

the signaling of abiotic stress responsiveness. Among these genes, $V v N F-Y B 18$ responded to various stresses and showed different expression patterns upon these treatments, suggesting its different roles in multiple signaling pathways.

We next analyzed the possible involvement of NF-Y genes in response to biotic stresses. No obvious changes
( $\geq 1$-fold) in expression levels of $V v N F-Y s$ were detected in 'Cabernet Sauvignon' after powdery mildew fungus (PM) infection (data not shown), and this result is consistent with a previous report [37]. The expression patterns of VvNF-Ys after Plasmopara viticola inoculation were also analyzed. Three $V v N F-Y B s$ were found to respond $(\geq 2$ fold) to P. viticola infection (Fig. 4). VvNF-YB13 and 


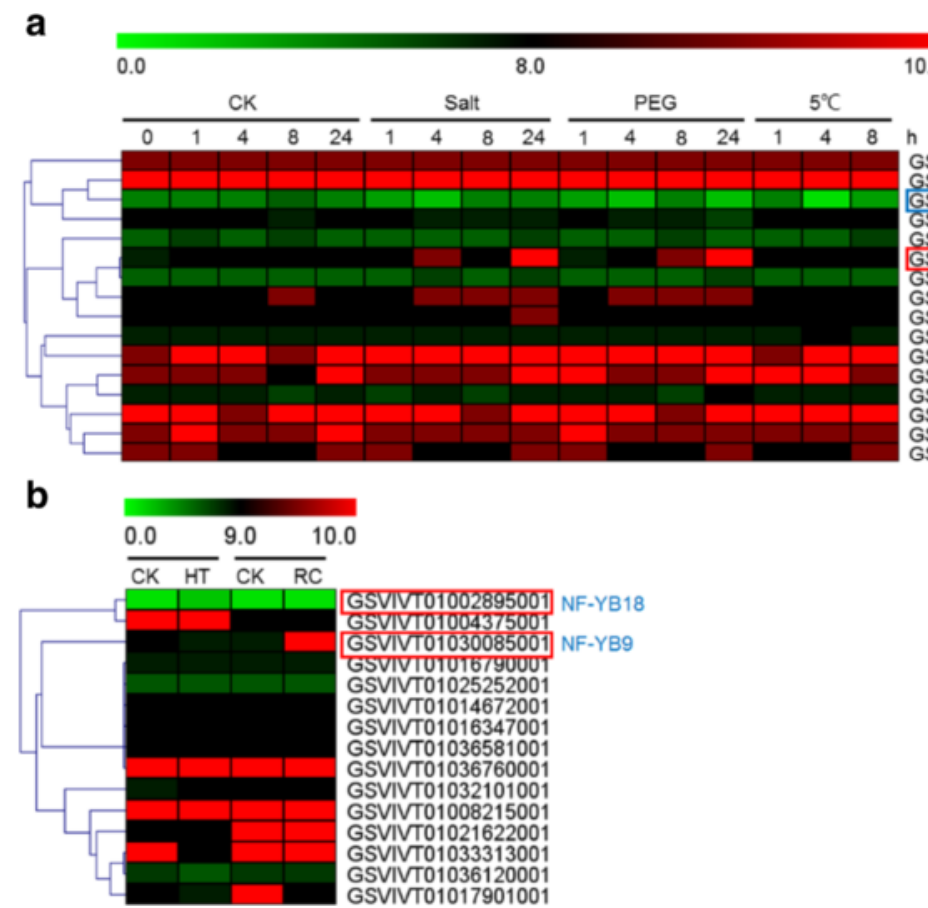

10.0

Fig. 3 Expression profiles of VVNF-YS in response to abiotic stress treatments. a Expression patterns of VVNF-Ys in response to salt, drought (PEG) and cold $\left(5^{\circ} \mathrm{C}\right)$ treatments. $\mathbf{b}$ Expression patterns of VVNF-YS under heat stress treatment. HT represents high temperature and RC means recovery process. The color scale indicates fold-change values (log2 values) with red representing increased transcript abundance and green indicating decreased transcript abundance. A red box indicates up-regulation and blue box indicates down-regulation

$V v N F-Y B 17$ were down-regulated in incompatible plant bearing the resistant gene Rpv2 after $P$. viticola infection as compared to the mock control. The expression level of $V v N F-Y B 18$ in resistant plants (bearing Rpv1 or Rpv2) was increased after inoculation (Fig. 4). These results indicated that a certain number of $V v N F-Y s$ displayed pathogenrelated expression patterns, implying their possible involvement in grape immune signaling. Those $V v N F-Y S$ with altered expression levels might be candidates for further study of grape immune response.

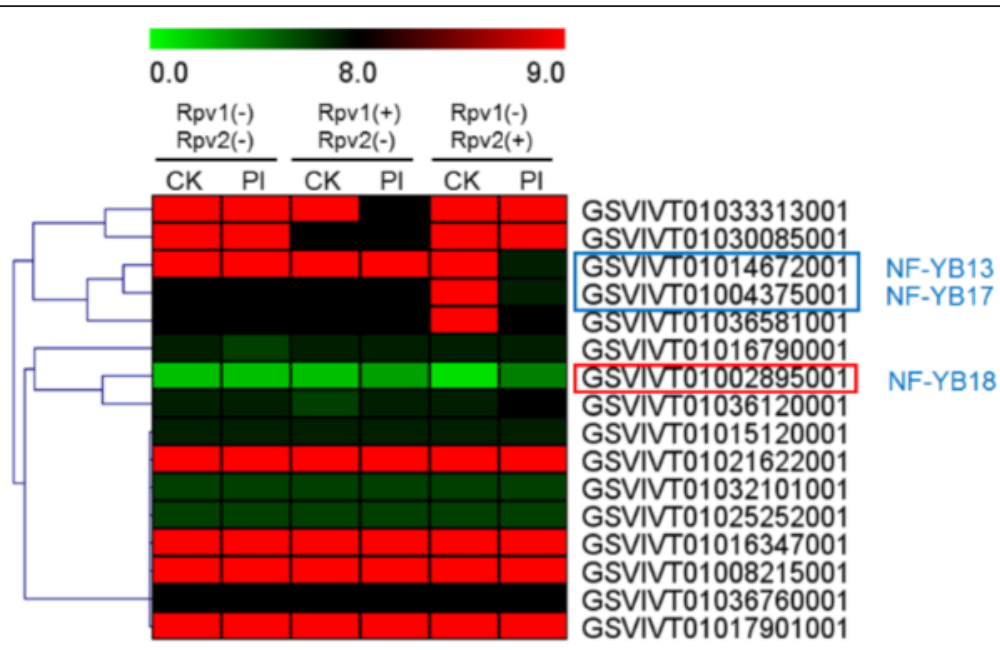

Fig. 4 Expression patterns of $V W N F-Y_{S}$ during downy mildew infection. Plants of different genotypes were used in the experiment: susceptible plants without resistance loci Rpv1 and Rpv2 (Rpv1 (-) Rpv2 (-)) and incompatible plants bearing the resistance gene Rpv1 (Rpv1 (+) Rpv2 (-)) or Rpv2 (Rpv1 (-) Rpv2 (+)). Pl means Plasmopara viticola inoculation. The color scale indicates fold-change values (log2 values) with red representing increased transcript abundance and green indicating decreased transcript abundance. A red box indicates up-regulation and blue box indicates down-regulation 
Phytohormones such as abscisic acid (ABA), methyl jasmonate (MJ), salicylic acid (SA), and ethylene have been reported to act as messengers in plant response to abiotic and biotic stresses [38]. To examine the influence of phytohormones on $V v N F-Y s$ expression, we analyzed the transcript levels of $V v N F-Y_{S}$ in 'Monastrell' ( $V$. vinifera) calli in response to exogenously applied $\mathrm{MJ}$ and cyclodextrin elicitor (CD). As shown in Fig. 5a, VvNF-YA7 showed a decreased transcript level ( $\geq 1$-fold) whereas $V v N F-Y A 8$ showed an increased transcript level $(\geq 1$-fold $)$ in response to exogenous $\mathrm{MJ}$ and $\mathrm{CD}$. For ABA treatment, grapevines of 'Cabernet Sauvignon' at veraison were treated with $400 \mathrm{mg} / \mathrm{L}$ ABA solution. Two $V v N F$ $Y s$ (1 $V v N F-Y A$ and $1 V v N F-Y B)$ showed a decrease in transcript abundance upon exogenous ABA treatment (Fig. 5b). In contrast to treatments with $\mathrm{MJ}$ and $\mathrm{CD}$, $V v N F-Y A 8$ was slightly down-regulated (0.5-fold) upon ABA treatment at 28 day after veraison (dav). The expression of VvNF-YB18, however, was repressed ( $>1$ fold) by exogenous ABA at 14 dav. The possible function of $V v N F-Y B 18$ in ABA signaling pathway may partly account for its involvement in response to abiotic and biotic stresses.

\section{Expression profiles of $V v N F-Y s$ in grape leaves and berries at different development stages}

To investigate expression patterns of $V v N F-Y s$ in two of the most important grape organs, leaves and berries, quantitative real-time PCR (qPCR) was conducted to analyze $V v N F-Y$ expression in leaves (L) and berries (F) of 'Semillon' $(V$. vinifera) at veraison $(\mathrm{V})$ and fully ripe stage (R) (namely, LV, LR, FV and FR), respectively. However, due to the homogeneous properties of $V v N F$ $Y s$ as well as the fact that primers did not work as well as expected, about half of the $V v N F-Y s$ were selected for
qPCR analyses, and 13 yielded significant results (Fig. 6a). Generally, the expression levels of $V v N F-Y s$ in leaves were higher $(P<0.01)$ than that in berries, and most of $V v N F-Y s$ exhibited higher transcript levels $(P<0.05)$ in LV than in LR (Fig. 6a). For example, VvNF-YB8 was differentially expressed $(P<0.01)$ in leaves, and the transcript level of $V v N F-Y B 8$ was much higher $(P<0.01)$ in LV as compared to that in LR (Fig. 6a). This result indicated that $V v N F-Y B 8$ might be a tissue-specific gene and participate in synthesis-oriented biological processes. It is notable that the transcript abundance of most $V v N F$ $Y$ s exhibited no much difference in berries with the exception being $V v N F-Y C 5$, which had a higher expression level $(P<0.05)$ in FR rather than in FV (Fig. 6a). All these results suggested that some of $V v N F-Y s$ may be associated with grape development, which is consistent with the results of RNA-seq (data not shown). qPCR was conducted to further demonstrate the expression patterns of $V v N F-Y_{S}$ in grape berries at three different developmental stages. More than half of tested $V v N F-Y_{S}$ were predominantly expressed $(P<0.01)$ in specific development period as expected (Fig. 6b). VvNF-YA7 and $V v N F-Y B 14$ were differentially expressed at fruit set (FS), while $V v N F-Y B 4$ and $V v N F-Y B 8$ were dominantly expressed at veraison $(\mathrm{V})$. The transcript level of $V v N F$ $Y B 8$ was decreased $(P<0.01)$ at the development stage of fully ripe (R). These results indicated that $V v N F-Y s$ might paly roles throughout the development of grape, and expression of specific genes would be regulated at certain stages of grape development.

Additionally, expression profiles of $V v N F-Y_{S}$ in different berry tissues were also detected [39]. Five $V v N F-Y s$ exhibited different expression patterns in different berry tissues (Fig. 6c). Two VvNF-YAs (VvNF-YA3 and VvNFF$Y A 8)$ and $V v N F-Y B 13$ were differentially expressed $(>2-$
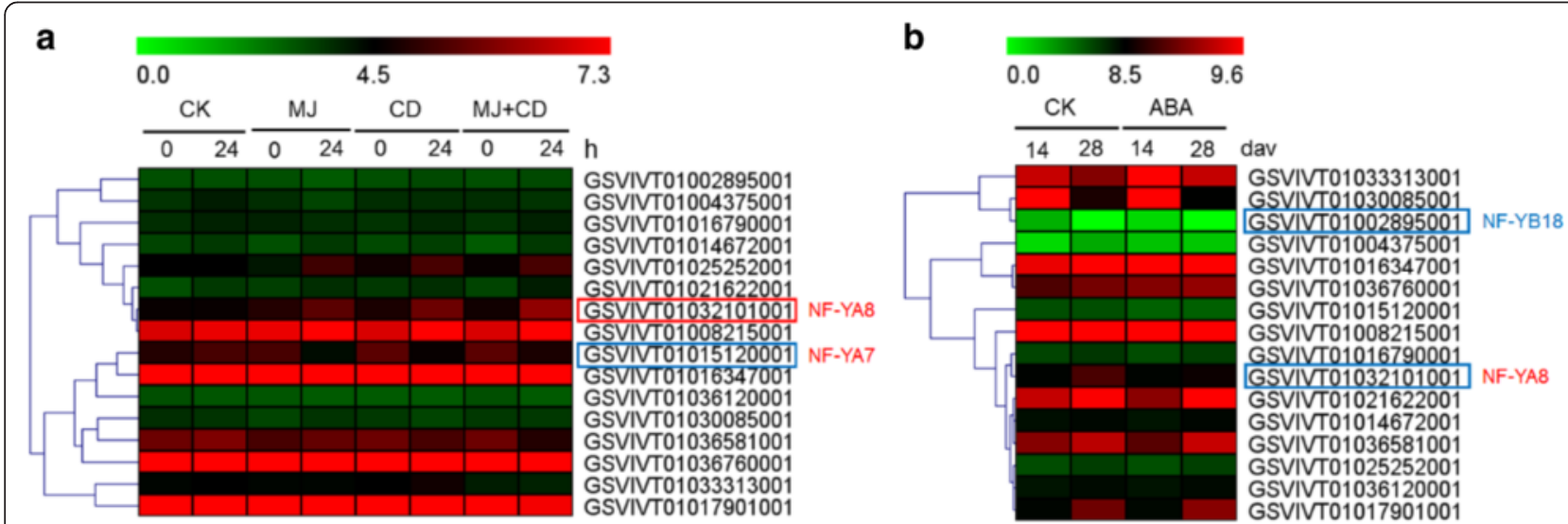

Fig. 5 Expression patterns of VVNF-YS in response to phytohormone and elicitor treatment. a Expression patterns of $V_{V N F}-Y_{S}$ in response to methyl jasmonate (MJ) and cyclodextrin elicitor (CD). b Expression patterns of VVNF-Y $Y_{S}$ under ABA treatment. Dav represents days after veraison. The color scale indicates fold-change values (log2 values) with red representing increased transcript abundance and green indicating decreased transcript abundance. A red box indicates up-regulation and blue box indicates down-regulation 


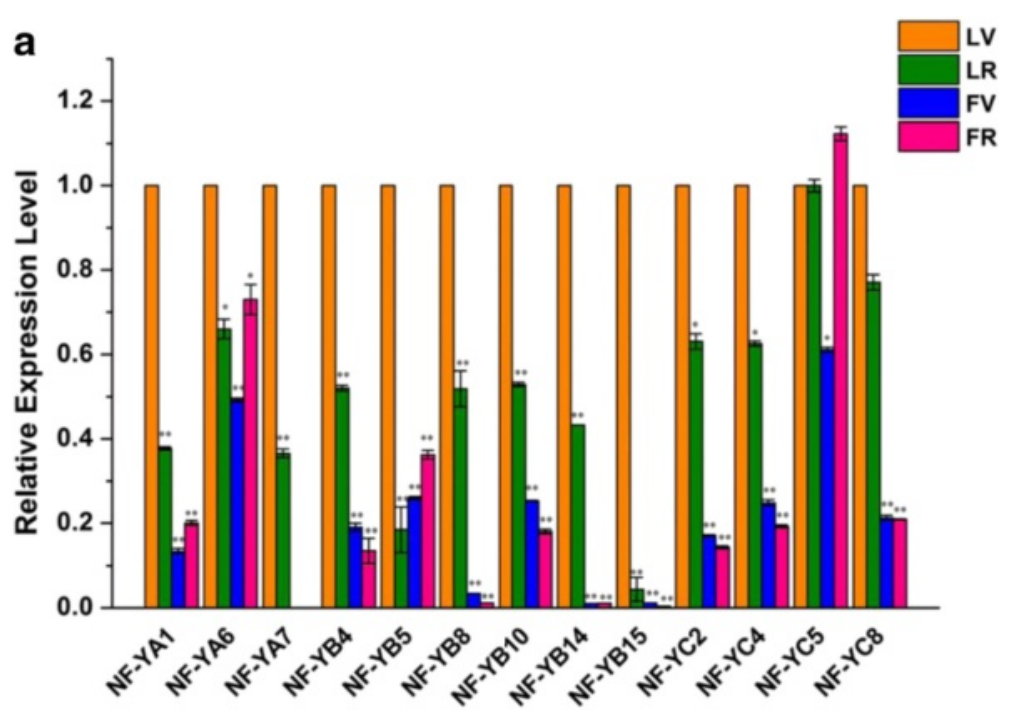

b

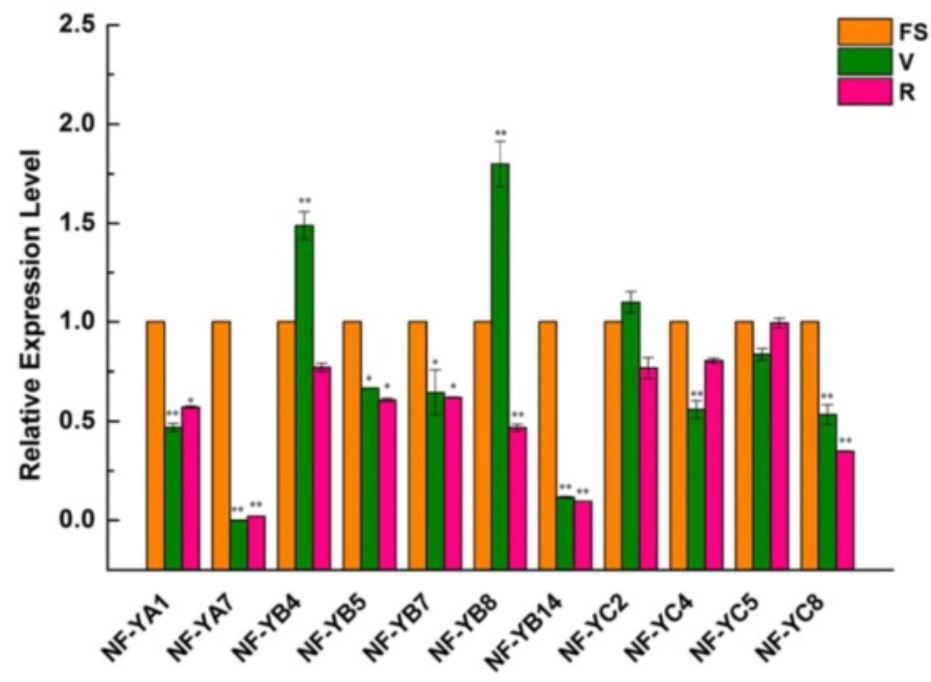

c
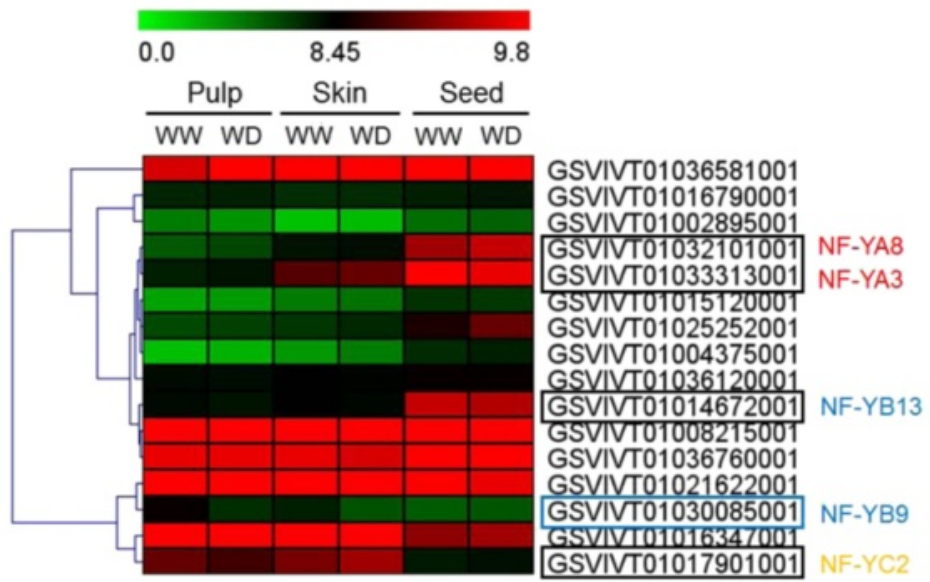

Fig. 6 (See legend on next page.) 
(See figure on previous page.)

Fig. 6 Expression profiles of $V_{V N F-Y S}$ in grape leaves and berries at different developmental stages. a Detailed expression levels of $V_{V} N F-Y_{S}$ in grape leaves and berries. LV and LR denote leaves at veraison ( $V$ and fully ripe (R) while FV and FR represent berries at $V$ and $R$, respectively. $\mathbf{b}$ Detailed expression levels of VVNF-YS in grape berries at three different developmental stages: fruit set (FS), $V$ and R. c Expression patterns of $V v N F-Y_{S}$ in different berry tissues. WW and WD mean well-watered and water-deficit conditions, respectively. Grape Actin 1and UBC were used as internal controls of quantitative real-time PCR analysis. The data are presented as mean values \pm SD. ${ }^{*}$ and ${ }^{* *}$ represent statistically significant $(P<0.05)$ and highly significant $(P<0.01)$ differences, respectively. Significance of values in $(\mathbf{a})$ was based on comparison of expression levels in leaves and berries at different stages with expression levels in leaves at $\mathrm{V}$ while in (b) was based on comparison of expression levels in berries at $\mathrm{V}$ and $\mathrm{R}$ with expression levels in berries at FS

fold) in seed, and $V v N F-Y B 9$ as well as $V v N F-Y C 2$ was predominantly expressed $(>1.5$-fold) in pulp and skin (Fig. 6c). Notably, the expression of $V v N F-Y B 9$ in pulp and skin was affected ( $>1.5$-fold) by water supply, suggesting its possible role in response to water deficiency.

\section{Expression of $V v N F-Y_{s}$ in response to different sugar content and exogenous sugar treatment}

The expression levels of some $V v N F-Y s$ were increased in grape berries at veraison, which is characterized by the accumulation of hexose sugar in flesh and skin [40]. To examine whether there exists a relationship between expression of $V v N F-Y s$ and sugar content, we first investigated the expression levels of $V v N F-Y s$ in five grape varieties with different sugar contents (Fig. 7). The content of glucose, sucrose and fructose was measured, respectively, and sucrose was omitted from the analysis because of its extremely low content. Besides, the content of glucose was close to that of fructose (data not shown), so fructose and total sugar were finally chosen to evaluate the correlation between sugar content and $V v N F-Y s$ expression. There were four $V v N F-Y s$ (1 $V v N F-Y A, 1 V v N F-Y B$ and $2 V v N F-Y C s)$ changing their expression with the contents of fructose in all varieties. Interestingly, variation of expression levels of most $V v N F-Y s$ was consistent with that of fructose contents

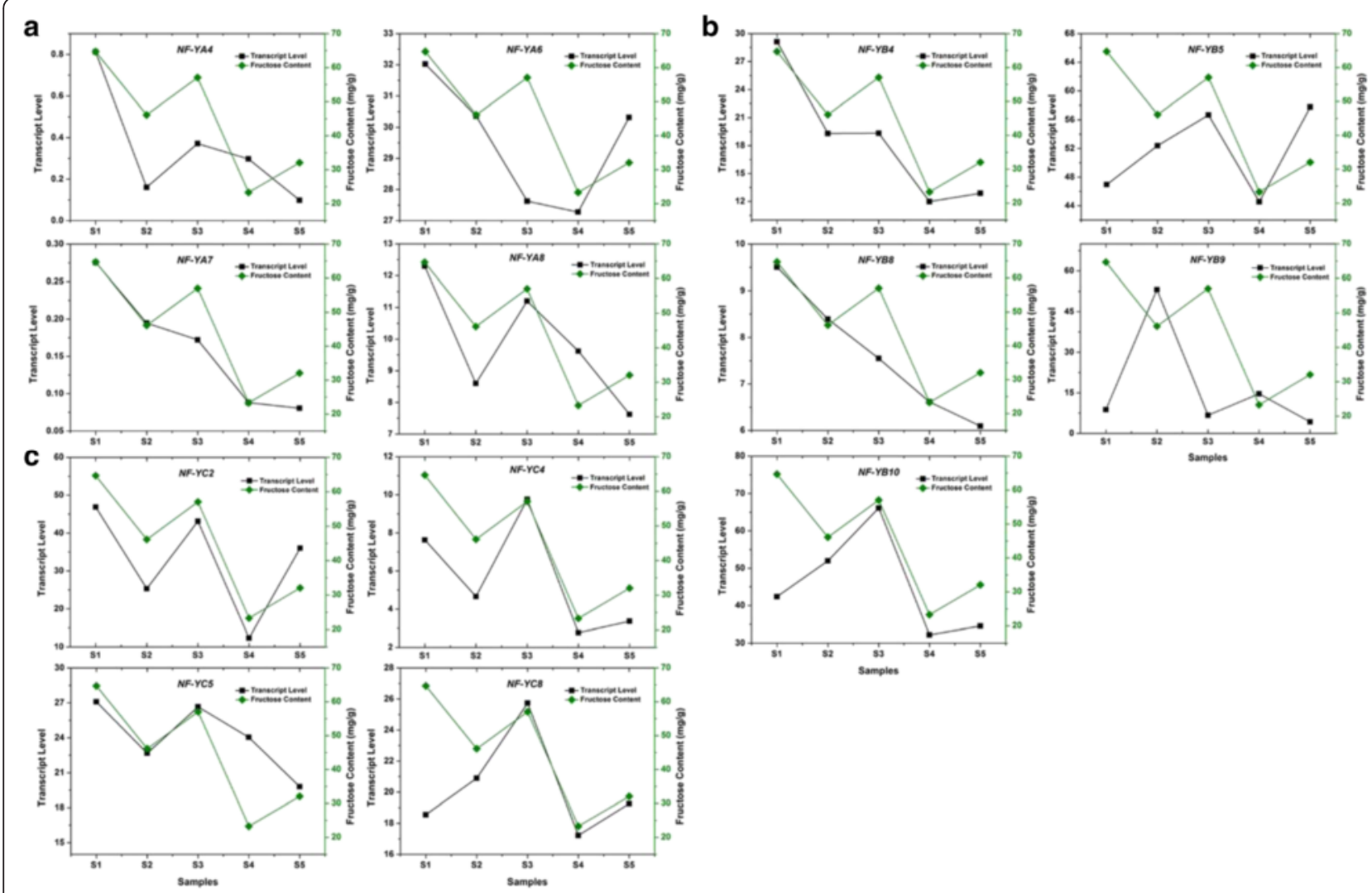

Fig. 7 Expression levels of VVNF-Ys and fructose content in different grape varieties. VVNF-YAs (a) VVNF-YBs (b) and VVNF-YCS (c) are divided into three groups. Soluble sugars were extracted from five grape varieties (S1-S5) and then analyzed by HPLC with water as eluent. The fructose content was relatively stable in three successive years (unpublished data), so values for 1 year are given as reference. Black broken lines denote transcript levels of NF-Y genes, and green broken lines indicate fructose content in grape berries 
in at least four grape varieties (Fig. 7). However, this sugar-related expression pattern of $V v N F-Y s$ no longer exist if examined with total sugar contents (Additional file 4: Figure S3). These results suggested that a number of $V v N F-Y s$, such as $V v N F-Y A 7, V v N F-Y B 4$ and $V v N F-$ $Y C 2$, may tend to be responsive to specific sugars.

To verify the hypothesis that some VvNF-Ys could respond to specific sugar, we analyzed the expression patterns of $V v N F-Y s$ in 'Chardonnay' ( $V$. vinifera) suspension cells after treatment with exogenous glucose, sucrose and fructose at a final concentration of 0.0 (CK), 0.5, 1.0, or $2.0 \%(\mathrm{w} / \mathrm{v})$, respectively. In general, all the tested $V v N F-Y_{S}$ could be induced $(P<0.01)$ by exogenous fructose except for $V v N F-Y C 8$ (Fig. 8), and most $V v N F-Y s$ were downregulated $(P<0.05)$ in response to glucose and sucrose treatments (Fig. 8). The expression of $V v N F-Y A 1$ and $V v N F-Y B 7$, however, was induced $(P<0.01)$ by sucrose treatment (Fig. 8a-b). Additionally, the expression of $V v N F-Y B 7$ was also induced by glucose (Fig. $8 \mathrm{~b}$ ). Intriguingly, 11 out of $14 V v N F-Y s$ responded strongly to the fructose treatment at the final concentration of $0.5 \%$ with their transcript levels increasing from 1.3-fold $(V v N F-Y C 8)$ to more than 10-fold ( $V v N F-Y A 6)$. Nevertheless, some of them, i.e. $V v N F-Y A 7, V v N F-Y B 14$, $V v N F-Y B 15$ and $V v N F-Y C 6$, exhibited decreased transcript levels $(P<0.05)$ at high concentration $(1.0$ and $2.0 \%$ ) of fructose (Fig. 8). These data showed that a reasonable number of $V v N F-Y s$ displayed sugar-responsive expression pattern and their expression may be affected by the kind and concentration of exogenous sugars. The two VvNF-Ys (VvNF-YA1 and VvNF-YB7) induced by sucrose could be involved in the biosynthesis and/ or transport of sucrose in grape. The expression of $V v N F-Y A 1$ and $V v N F-Y B 7$ was promoted after exposure to fructose and glucose, respectively, indicating their potential roles in accumulation of these two soluble sugars during grape ripening.

\section{Discussion}

NF-Y proteins have been revealed to be key factors in multiple physiological processes in plants $[12,13,24,25$, $41,42]$. However, the function of most NF-Y proteins in grape are still unknown. Here we tried to take advantage of available data to explore and analyze the grape $N F-Y$ family. Based on the results obtained from searching the Uniprot database by using PFAM and KOG IDs of conserved domains, we identified 32 previously predicted NF-Y proteins in PlantTFDB and two more members (Table 1). The two proteins, VvNF-YA1 and VvNF-YB13, consisted of more than 1000 amino acids, and their corresponding genes contained 13 and 11 exons, respectively (Additional file 2: Figure S2). Sequence analysis in InterPro revealed that VvNF-YA1 contained multiple functional domains, and the CBF signature (CBFB/NFYA,
PFAM02045) was located between glutamic $\left(\mathrm{E}_{1510}\right)$ and phenylalanine $\left(\mathrm{F}_{1572}\right)$ next to the N-terminus. VvNF-YB13 contained only two domains, and the CBF domain (CBFD_NFYB_HMF, PF00808) was located between arginine $\left(R_{914}\right)$ and methionine $\left(M_{979}\right)$ close to the $\mathrm{N}$ terminus as well. The phylogenetic analysis showed that VvNF-YA1 was homologous to VvNF-YA6, AtNF-YA4 and AtNF-YA7, while VvNF-YB13 was homologous to VvNF-YB14, AtNF-YB2 and AtNF-YB3 (Fig. 1). Altogether, VvNF-YA1 and VvNF-YB13 were regarded as the members of grape NF-Y family.

Evolutionary analysis could be used to predict potential functions of unknown members based on the known functions of those well-studied members of the same clade [3, 43, 44]. Therefore, an un-rooted phylogenetic tree based on sequences of NF-Y proteins from grape and Arabidopsis were constructed to explore the functions of VvNF-Ys (Fig. 1). For example, AtNF-YB2 and $A t N F-Y B 3$, the homologous genes of $V v N F-Y B 13$ and $V v N F-Y B 14$ in Arabidopsis, were reported to promote flowering in response to inductive long-day condition [45], so the two $V v N F-Y B s$ might play roles in regulation of flowering in grape. More importantly, the genes required for flowering time control were generally expressed in leaf vascular tissue $[45,46]$, and our data showed that $V v N F-Y B 14$ was differentially expressed in leaves (Fig. 6a). This result indicates that VvNF-YB14 should be the candidate gene of particular interest for further study of flowering time control in grape.

Alterations in exon-intron structure or conserved domains would change the function of the gene or protein $[1,47]$. Analysis of exon-intron structures revealed that most of $V v N F-Y A s$ had similar exon-intron organization pattern whereas $V v N F-Y B s$ and $V v N F-Y C s$ exhibited more variable and complicated structures (Additional file 2: Figure S2). Multiple alignments of NF-Y protein sequences among different species revealed the conservation of functional domains in VvNF-YAs (Fig. 2a). VvNF-YBs and VvNF-YCs, however, exhibit alterations in their functional domains, yet most of functionality required residues are conserved (Fig. 2b, c). The conservation of functional residues indicates the conserved functions of VVNF-Ys as their orthologs function in other plant lineages [47-52], whereas changes in protein sequences may imply the alterations of function. A good example is that $V v L 1 L$, the homolog of $A t L 1 L$ in grape, has been reported to play a role in grape somatic embryogenesis [24, 25].

To further investigate the function of VvNF-Ys, the expression patterns of $V v N F-Y s$ in different grape organs and in response to various stresses were examined. Some of $V v N F-Y s$ were found to respond to at least one kind of stress treatments. For instance, $V v N F-Y A 3$ and $V v N F$ $Y B 18$ were apparently induced by salt and PEG treatments 


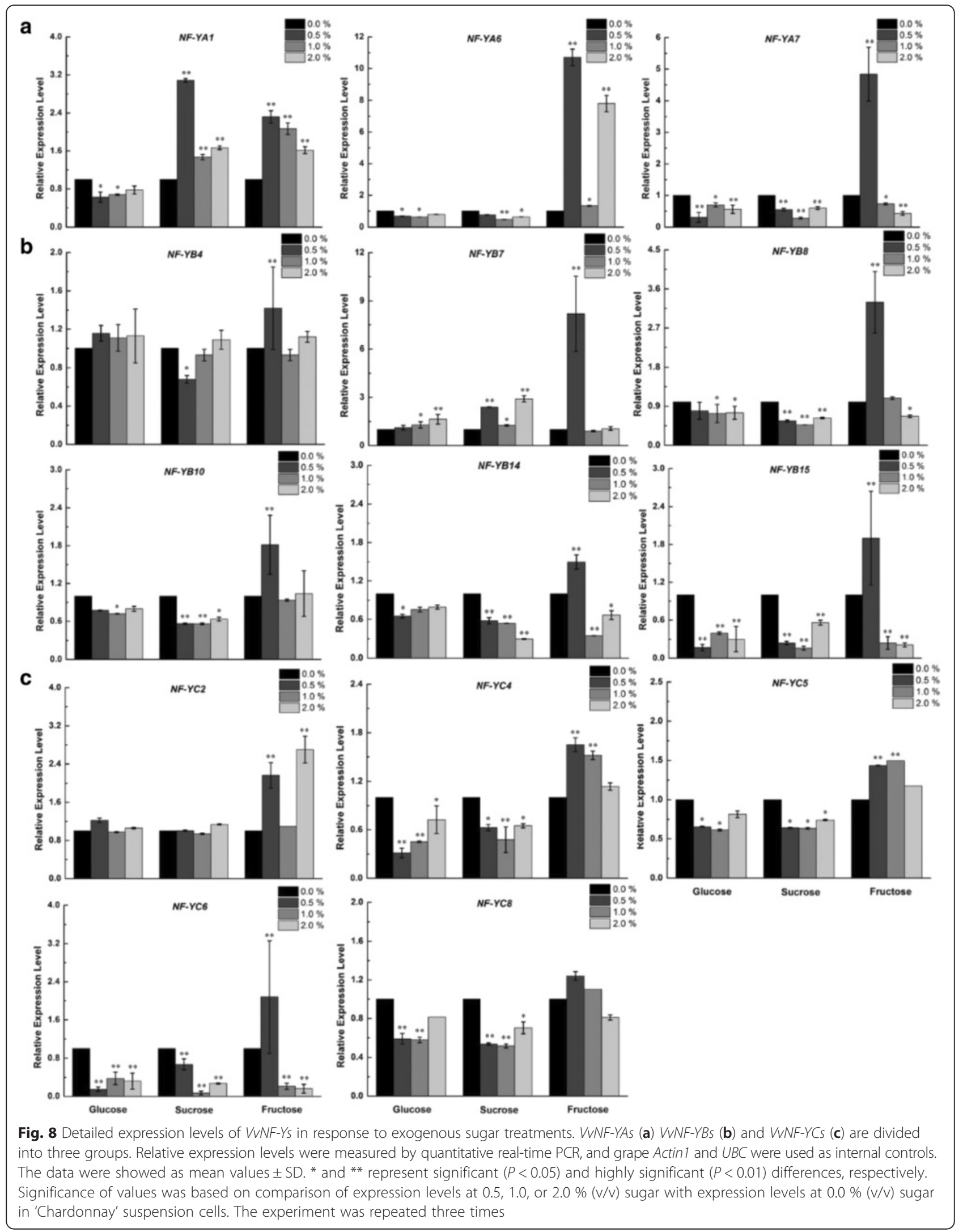


(Fig. 3a and Table 2). VvNF-YA3 was homologous to AtNF-YA1 (Fig. 1), which was previously reported to function in seed development and could be induced by drought treatment $[2,49,53]$. Given that $V v N F-Y A 3$ was mainly expressed in seed (Fig. 6c), it can help improve the resistance of grape seed to water deficiency. In addition, $V v N F-Y A 3$ might be also involved in maturation of seed and dehydration signaling [53]. Intriguingly, $V v N F-Y B 18$ was also revealed to respond to heat, PI and ABA treatments (Table 2). The results showed that some $V v N F-Y s$ might be regulators of multiple biological processes. A certain number of $V v N F-Y_{S}$ were revealed to be involved in response to certain stress treatment. The expression of $V v N F-Y B 9$ was down-regulated after the heat treatment but increased again during the following recovery process (Fig. 3a and Table 2). Interestingly, $V v N F-Y B 9$ was predominantly expressed (>1.5-fold) in pulp and skin (Fig. 6c), suggesting a role in grape berries in response to environmental temperature stress. $V v N F-Y B 13$ and $V v N F-Y B 17$ only responded to PI and their expression seems to be genotype-dependent (Fig. 4 and Table 2). Phytohormone treatments showed that $V v N F-Y A 8$ could respond to various hormones with increased transcript level in response to $\mathrm{MJ}$ and $\mathrm{CD}$, and decreased transcript level to ABA (Fig. 5 and Table 2). The results indicated that $V v N F-Y A 8$ might play different roles in different hormone signaling pathways. Emerging evidence suggests

Table 2 Expression patterns of VVNF-Ys in response to specific stresses

\begin{tabular}{lll}
\hline Gene Name & Stress response & Expression pattern \\
\hline NF-YA1 & Sucrose, fructose & Up-regulated \\
NF-YA3 & Salt, PEG & Up-regulated \\
NF-YA6 & Fructose (2.0\%) & Up-regulated \\
NF-YA7 & MJ, CD & Down-regulated \\
& Glucose, sucrose & Down-regulated \\
& Fructose (0.5 \%) & Up-regulated \\
NF-YA8 & MJ, CD & Up-regulated \\
& ABA & Down-regulated \\
NF-YB9 & Heat stress & Down-regulated \\
& Recovery process after heat stress & Up-regulated \\
NF-YB13 & Plasmopara viticola infection & Down-regulated \\
& (with plant bearing Rpv2) & \\
NF-YB17 & Plasmopara viticola infection & Down-regulated \\
& (with plant bearing Rpv2) & \\
NF-YB18 & Salt, PEG, cold (5 ${ }^{\circ}$ C) & Down-regulated \\
& Heat stress & Up-regulated \\
& Plasmopara viticola infection & Up-regulated \\
& (with plant bearing Rpv1 or Rpv2) & \\
& ABA & Down-regulated \\
& Fructose (2.0 \%) & Up-regulated \\
\hline
\end{tabular}

that hormone signaling pathways regulated by $\mathrm{ABA}$ and MJ play significant roles in the crosstalk between abiotic and biotic stress signaling [54]. VvNF-YA8, along with $V v N F-Y B 18$ discussed above, could be proposed as promising candidate that involved in crosstalk between hormone and stress signaling pathways.

A common response of plants to abiotic stresses such as drought and salinity is the accumulation of sugars and other compatible solutes $[55,56]$. The study of relationship between expression levels of $V v N F-Y s$ and sugar contents showed that the changes of fructose contents and expression levels of the tested $V v N F-Y s$ were highly consistent in at least four individual grape varieties (Fig. 7). Exogenous sugar treatments showed that expression of most $V v N F-Y s$ were down-regulated in response to exogenous glucose and sucrose treatments (Fig. 8), except that $V v N F-Y B 7$ was up-regulated by glucose (the peak was around 2.0-fold, $p<0.01$ ) and sucrose (the peak was over 3.0 -fold, $p<0.01$ ). This suggested that $V v N F-Y B 7$ could be involved in carbohydrate anabolism in grape. Notably, almost all the $V v N F$ $Y s$ responded strongly to exogenous fructose treatment at the concentration of $0.5 \%$ (Fig. 8). Among these genes, $V v N F-Y C 2$ and $V v N F-Y A 6$ responded to a higher concentration $(2.0 \%)$ of fructose likewise (Table 2), implying that they might play roles in fructose accumulation in grape berries ripening. Nevertheless, the expression of $V v N F-Y A 6$ was suppressed by exogenous glucose and sucrose. All the results revealed that the regulatory networks of sugar accumulation or metabolism in grape are complicated, and the involvement of $V v N F-Y s$ in grape berry sugar signaling still need more experimental evidence.

\section{Conclusions}

In the present study, $34 V v N F-Y s$ were identified, and evolutionary, structural and expression analyses were conducted to reveal their possible biological roles in stress responses, development, and sugar metabolism. Comparison of NF-Ys from grape and Arabidopsis provided rudimentary insight on the function of lessstudied $V v N F-Y s$ in relation to their well-understood homologs. Furthermore, investigation of expression profiles showed that $V v N F-Y s$ responded to various abiotic and biotic stresses as well as hormone treatments. Moreover, analysis of $V v N F-Y s$ expression during grape berry development revealed that $V v N F-Y S$ might play roles in fruit set, ripening and sugar accumulation in grape berry. Based on prediction and experimental data, the $V v N F-Y_{s}$ might be involved in responses to salt, drought, cold and pathogens and may also play significant roles in grape berry development as well as sugar accumulation. More significantly, $V v N F-Y s$ probably function as regulators to mediate cross-talk between different 
signaling pathways. All these results may contribute to further functional investigation of grape $N F-Y$ family.

\section{Methods \\ Identification of VvNF-Y genes}

The sequences of grape NF-Y proteins were obtained from the UniProt (http://www.uniprot.org/), using PFAM ID PF02045, PF00808 and KOG ID KOG0869, KOG0871, KOG1561 for NF-YA (HAP2), NF-YB (HAP3) and NF-YC (HAP5), as queries [2]. The obtained sequences were then compared with those from the PlantTFDB database v3.0 (http://planttfdb.cbi.pku.edu.cn/) [57]. All putative NF-Y proteins were further verified with the tool of InterProScan (http://www.ebi.ac.uk/Tools/pfa/iprscan/) to confirm the existence of the core domains. The corresponding sequences of $N F-Y$ genes were acquired from the Grape Genome Browser (12×) (http://www.genoscope.cns.fr/ externe/GenomeBrowser/Vitis/). The incomplete and redundant sequences were omitted.

\section{Structure and chromosomal localization}

The locations of NF-Y genes on grape chromosomes were obtained from the Grape Genome Browser $(12 \times)$ (http://www.genoscope.cns.fr/externe/GenomeBrowser/ Vitis/). Gene structures (exon-intron structures) were visualized by alignment of cDNA sequences with corresponding genomic DNA sequences with the online tool of GSDS 2.0 (http://gsds.cbi.pku.edu.cn/).

\section{Alignments and phylogenetic analysis of NF- $Y$ genes}

Multiple sequence alignments of identified grape and Arabidopsis NF-Y proteins were conducted using ClustalX 2.0 [58]. Then the results were used to construct Neighbor-Joining tree using MEGA5.0 with the number of bootstrap replications being set at 1000 [59]. The sequences of Arabidopsis NF-Y proteins used for analyses were obtained from the PlantTFDB database v3.0 (http:// planttfdb.cbi.pku.edu.cn/) and the Arabidopsis Information Resource (TAIR, https://www.arabidopsis.org/).

\section{Plant materials and sugar treatment}

Grape (V. vinifera) seedlings were grown in the Vitis germplasm resources garden of Institute of Botany, the Chinese Academy of Sciences, Beijing, under natural conditions. For qPCR analysis of NF-Ys expression, grape berries and leaves at fruit set, veraison and fully ripe stages were sampled and ground into powder in liquid nitrogen before RNA extraction.

Grape suspension cells derived from embryogenic callus that was induced from whole flowers of 'Chardonnay' grape were used for exogenous sugar treatment. The suspension cells were cultured in $250 \mathrm{~mL}$ flasks filled with $50 \mathrm{~mL}$ of liquid CSM medium (MS basal medium supplemented with $0.5 \mathrm{~g} / \mathrm{L}$ glutamic acid, $1 \mathrm{mg} / \mathrm{L}$ NOA, $5.0 \mathrm{~mL} /$
$\mathrm{L}$ glycerol and $20 \mathrm{~g} / \mathrm{L}$ maltose), and shaken at $117 \mathrm{rpm}$ at $27 \pm 1{ }^{\circ} \mathrm{C}$ in the dark. All the suspension cells were subcultured every 7 days. For exogenous sugar treatment, glucose, sucrose and fructose were added to the media for final concentrations of $0.5,1$, and $2 \%(\mathrm{w} / \mathrm{v})$, respectively, at the time of $5 \sim 6$ days (logarithmic growth phase of cells) after subculture. Then, the suspension cultures were centrifuged at $5000 \mathrm{rpm}$ for $5 \mathrm{~min}$, and the liquid media was removed. The collected cells were washed $3 \sim 4$ times with sterile deionized water and subsequently ground in liquid nitrogen for RNA extraction. Each treatment replicated three times.

\section{RNA isolation and quantitative real-time PCR}

Total RNA was extracted using the RNAprep Pure Plant Kit (TianGen, Beijing, China) according to the manufacturer's instructions. RNA integrity was confirmed by electrophoresis on $1 \%$ agarose gels, and concentration as well as quality of RNA were detected by NanoDrop 2000 Spectrophotometer (Thermo Scientific, MA, USA). The removal of genomic DNA and synthesis of the first strand cDNA was performed using HiScript Q RT SuperMix for qPCR (+ gDNA wiper) Kit (Vazyme, Nanjing, China). Quantitative real-time PCR (qPCR) was carried out using AceQ qPCR SYBR Green Master Mix (Vazyme, Nanjing, China) with the CFX96 System (BioRad, CA, USA). The qPCR reactions consisted of a hold at $95{ }^{\circ} \mathrm{C}$ for $5 \mathrm{~min}$, followed by 40 cycles at $95{ }^{\circ} \mathrm{C}$ for $10 \mathrm{~s}$ and $60{ }^{\circ} \mathrm{C}$ for $30 \mathrm{~s}$. Melting curve was included to verify the specificity of each primer pair. Grape Actin1 (accession no. AY680701) and $U B C$ (accession no. EC922622) [60] were used as internal controls. The results were evaluated by the method of the $2^{-\Delta \Delta C t}$ [61] The data were obtained from three technological and biological replicates and are shown as mean values \pm SD. The significance of differential expression between controls and treatments was examined by Student's $t$-test with $P<0.05$ and $P<0.01$ indicating statistically significant and highly significant, respectively. Primers used for qPCR are listed in Additional file 5: Table S2.

\section{Microarray and transcriptome data analysis}

Two databases, the ViTis Co-expression DataBase (VTCdb, http://vtcdb.adelaide.edu.au/Home.aspx) [62] and the Plant Expression Database (PLEXdb, http:// www.plexdb.org/index.php), were searched for the probe sets of grape $N F-Y$ genes. Finally, 16 probe sets, which were designed for Affymetrix GeneChip $16 \mathrm{~K}$ Vitis vinifera (Grape) Genome Array and Affymetrix GrapeGen Vitis vinifera Array, were found in both two databases and successfully matched to the 16 sequences of the 34 identified $N F-Y$ genes (Additional file 3: Table S1).

Microarray data of grape $N F-Y$ genes were obtained from PLEXdb (http://www.plexdb.org/index.php). Probe 
IDs of NF-Y genes were used as query items to search the Affymetrix GeneChip platform. The expression data for selected NF-Y genes were obtained and shown as heatmaps with a color scale indicating $\log 2$ expression values.

\section{Sugar extraction and HPLC analysis}

Grape berries were collected and ground into powder in liquid nitrogen. The extraction of soluble sugars was conducted as previously described [63]. Around $100 \mathrm{mg}$ of powder was fitted with $10 \mathrm{~mL}$ of methanol:chloroform:water $(12 / 5 / 3 ; \mathrm{v} / \mathrm{v} / \mathrm{v})$, then sonicated for $30 \mathrm{~s}$, and subsequently centrifuged at $1200 \times \mathrm{g}$ for $10 \mathrm{~min}$. The supernatants were collected and diluted with water $(5 / 3$; $\mathrm{v} / \mathrm{v})$. Finally, $2 \mathrm{~mL}$ of the aqueous phase was evaporated and then dissolved in $0.8 \mathrm{~mL}$ of deionized water. The prepared samples were analyzed by HPLC with water as eluent $\left(0.6 \mathrm{~mL} \mathrm{~min}^{-1}\right)$.

\section{Additional files}

Additional file 1: Figure S1. Distribution of NF-Y genes on grape chromosomes. The NF-Y genes are ordered arranged according to their relative locations on chromosomes. (PDF $246 \mathrm{~kb}$ )

Additional file 2: Figure S2. Exon-intron structures of grape NF-Y genes. Rectangles indicate exons, and broken lines represent introns. (PDF $219 \mathrm{~kb}$ )

Additional file 3: Table S1. Probe sets from Affymetrix Microarray Platform for grape NF-Y genes used in microarray analysis following abiotic, biotic, and phytohormone treatments, and expression pattern assays in grape berry tissues. (PDF $100 \mathrm{~kb}$ )

Additional file 4: Figure S3. Expression levels of VVNF-YS and total sugar content in different grape varieties. VVNF-YAs (a), VVNF-YBs (b) and VVNF-YCS (c) are divided into three groups. Soluble sugars were extracted from five grape varieties (S1-S5) and then analyzed by HPLC with water as eluent. The total content was relatively stable in three successive years (unpublished data), so values for 1 year are given as reference. Black broken lines denote transcript levels of NF-Y genes, and blue broken lines indicate total sugar content in grape berries. (PDF $224 \mathrm{~kb}$ )

Additional file 5: Table S2. Primers for quantitative real-time PCR analysis of grape NF-Y genes expressions in this study. (PDF $96 \mathrm{~kb}$ )

\section{Abbreviations}

ABA, abscisic acid; CBF, CCAAT binding factor; CD, cyclodextrin elicitor; Dav, day after veraison; HAP, heme activator protein; MJ, methyl jasmonate; NF-Y, nuclear factor Y; PI, Plasmopara viticola inoculation; PM, powdery mildew; $\mathrm{QPCR}$, quantitative real-time polymerase chain reaction; TF, transcription factor

\section{Acknowledgements}

We would particularly wish to thank Prof. Douglas D. Archbold for the revise and feedback on the manuscript.

\section{Funding}

This work was supported by the National Natural Science Foundation of China (NO. 31572090).

\section{Availability of data and materials}

The data (sequence alignment and phylogenetic tree) used for phylogenetic analysis have been deposited in TreeBASE repository under the URL http:// purl.org/phylo/treebase/phylows/study/TB2:S19620. The microarray data used in this article are available in the Gene.

Expression Omnibus (GEO) repository under accession numbers of GSE31594 (http://www.ncbi.nlm.nih.gov/geo/query/acc.cgi?acc=GSE31594), GSE6404
(http://www.ncbi.nlm.nih.gov/geo/query/acc.cgi?acc=GSE6404), GSE31664 (http://www.ncbi.nlm.nih.gov/geo/query/acc.cgi?acc=GSE31664),GSE11406 (http://www.ncbi.nlm.nih.gov/geo/query/acc.cgi?acc=GSE11406),GSE42312 (http://www.ncbi.nlm.nih.gov/geo/query/acc.cgi?acc=GSE42312),GSE11857 (http://www.ncbi.nlm.nih.gov/geo/query/acc.cgi?acc=GSE11857), and GSE41423 (http://www.ncbi.nlm.nih.gov/geo/query/acc.cgi?acc=GSE41423), or are available at the PLEXdb (http://www.plexdb.org/modules/PD_browse/ experiment_browser.php).

\section{Authors' contributions}

$C R, S L$ and ZL designed the study. CR and YW performed bioinformatics analyses. ZZ carried out the treatments and HPLC analysis. CR performed qPCR tests and statistical analysis. CR draft the manuscript and ZL revised it. All authors read and approved the final manuscript.

\section{Competing interests \\ The authors declare that they have no competing interests.}

\section{Consent for publication}

Not applicable.

Ethics approval and consent to participate Not applicable.

\section{Author details}

${ }^{1}$ Beijing Key Laboratory of Grape Science and Enology and Key Laboratory of Plant Resource, Institute of Botany, the Chinese Academy of Sciences, Beijing 100093, People's Republic of China. ${ }^{2}$ University of Chinese Academy of Sciences, Beijing 100049, People's Republic of China.

Received: 9 May 2016 Accepted: 2 August 2016

Published online: 11 August 2016

\section{References}

1. Siefers N, Dang KK, Kumimoto RW, Bynum IV WE, Tayrose G, Holt III BF. Tissue-specific expression patterns of Arabidopsis NF-Y transcription factors suggest potential for extensive combinatorial complexity. Plant Physiol. 2009;149:625-41.

2. Quach TN, Nguyen HTM, Valliyodan B, Joshi T, Xu D, Nguyen HT. Genomewide expression analysis of soybean NF-Y genes reveals potential function in development and drought response. Mol Genet Genomics. 2015;290: 1095-115.

3. Li S, Li K, Ju Z, Cao DY, Fu DQ, Zhu HL, et al. Genome-wide analysis of tomato NF-Y factors and their role in fruit ripening. BMC Genomics. 2016;17:36.

4. Sinha S, Maity SN, Lu J, de Crombrugghe B. Recombinant rat CBF-C, the third subunit of CBF/NFY, allows formation of a protein-DNA complex with CBF-A and CBF-B and with yeast HAP2 and HAP3. Proc Natl Acad Sci U S A. 1995;92:1624-8.

5. Sinha S, Kim IS, Sohn KY, de Crombrugghe B, Maity SN. Three classes of mutations in the A subunit of the CCAAT-binding factor CBF delineate functional domains involved in the three-step assembly of the CBF-DNA complex. Mol Cell Biol. 1996;16:328-37.

6. Calvenzani V, Testoni B, Gusmaroli G, Lorenzo M, Gnesutta N, Petroni K, et al. Interactions and CCAAT-binding of Arabidopsis thaliana NF-Y subunits. PLoS One. 2012;7, e42902.

7. Frontini M, Imbriano C, Manni I, Mantovani R. Cell cycle regulation of NF-YC nuclear localization. Cell Cycle. 2004;3:217-22.

8. Kahle J, Baake M, Doenecke D, Albig W. Subunits of the heterotrimeric transcription factor NF-Y are imported into the nucleus by distinct pathways involving importin beta and importin 13. Mol Cell Biol. 2005;25:5339-54.

9. Mantovani R. The molecular biology of the CCAAT-binding factor NF-Y. Gene. 1999;239:15-27.

10. Riechmann JL, Heard J, Martin G, Reuber L, Jiang C, Keddie J, et al. Arabidopsis transcription factors: genome-wide comparative analysis among eukaryotes. Science. 2000;290:2105-10.

11. Petroni K, Kumimoto RW, Gnesutta N, Calvenzani V, Fornari M, Tonelli C, et al. The promiscuous life of plant NUCLEAR FACTOR Y transcription factors. Plant Cell. 2012;24:4777-92.

12. Braybrook SA, Harada JJ. LECs go crazy in embryo development. Trends Plant Sci. 2008;13:624-30. 
13. Gaj MD, Zhang S, Harada JJ, Lemaux PG. Leafy cotyledon genes are essential for induction of somatic embryogenesis of Arabidopsis. Planta. 2005;222:977-88,

14. Laloum T, De Mita S, Gamas P, Baudin M, Niebel A. CCAAT-box binding transcription factors in plants: Y so many? Trends Plant Sci. 2013;18:157-66.

15. Wenkel S, Turck F, Singer K, Gissot L, Gourrierec JL, Samach A, et al. CONSTANS and the CCAAT box binding complex share a functionally important domain and interact to regulate flowering of Arabidopsis. Plant Cell. 2006;18:2971-84.

16. Cao SH, Kumimoto RW, Gnesutta N, Calogero AM, Mantovani R, Holt III BF. A distal CCAAT/NUCLEARFACTORY complex promotes chromatin looping at the FLOWERINGLOCUST promoter and regulates the timing of flowering in Arabidopsis. Plant Cell. 2014;26:1009-17.

17. Combier JP, Frugier F, de Billy F, Boualem A, El-Yahyaoui F, Moreau S, et al. MtHAP2-1 is a key transcriptional regulator of symbiotic nodule development regulated by microRNA169 in Medicago truncatula. Genes Dev. 2006;20:3084-88.

18. Nelson DE, Repetti PP, Adams TR, Creelman RA, Wu JR, Warner DC, et al. Plant nuclear factor $Y$ (NF-Y) B subunits confer drought tolerance and lead to improved corn yields on water-limited acres. Proc Natl Acad Sci U S A. 2007;104:16450-5.

19. Li WX, Oono Y, Zhu J, He XJ, Wu JM, lida K, et al. The Arabidopsis NFYA5 transcription factor is regulated transcriptionally and posttranscriptionally to promote drought resistance. Plant Cell. 2008;20:2238-51.

20. West M, Yee KM, Danao J, Zimmerman JL, Fischer RL, Goldberg RB, et al. LEAFY COTYLEDON1 is an essential regulator of late embryogenesis and cotyledon identity in Arabidopsis. Plant Cell. 1994;6:1731-45.

21. Lotan T, Ohto M, Yee MK, West MA, Lo R, Kwong RW, et al. Arabidopsis LEAFY COTILEDON1 is sufficient to induce embryo development in vegetative cells. Cell. 1998;93:1195-205.

22. Liu JX, Srivastava R, Che P, Howell SH. An endoplasmic reticulum stress response in Arabidopsis is mediated by proteolytic processing and nuclear relocation of a membrane-associated transcription factor, bZIP28. Plant Cell. 2007;19:4111-99.

23. Liu JX, Howell SH. bZIP28 and NF-Y transcription factors are activated by ER stress and assemble into a transcriptional complex to regulate stress response genes in Arabidopsis. Plant Cell. 2010;22:782-96.

24. Schellenbaum $P$, Jacques $A$, Maillot $P$, Bertsch $C$, Mazet F, Farine $S$, et al. Characterization of VvSERK1, VvSERK2, VVSERK3 and VVL1L genes and their expression during somatic embryogenesis of grapevine (Vitis vinifera L.). Plant Cell Rep. 2008:27:1799-809.

25. Maillot $P$, Lebel $S$, Schellenbaum $P$, Jacques $A$, Walter B. Differential regulation of SERK, LEC1-Like and pathogenesis-related genes during indirect secondary somatic embryogenesis in grapevine. Plant Physiol Biochem. 2009;47:743-52.

26. Coustry F, Maity SN, Sinha S, de Crombrugghe B. The transcriptional activity of the CCAAT-binding factor CBF is mediated by two distinct activation domains, one in the CBF-B subunit and the other in the CBF-C subunit. J Biol Chem. 1996;271:14485-91.

27. Kim IS, Sinha S, de Crombrugghe B, Maity SN. Determination offunctional domains in the $C$ subunit of the CCAAT- binding factor (CBF)necessary for formation of a CBF-DNA complex: CBF- B interactssimultaneously with both the CBF-A and CBF-C subunits to form aheterotrimeric CBF molecule. Mol Cell Biol. 1996:16:4003-13.

28. Romier C, Cocchiarella F, Mantovani R, Moras D. The NF-YB/NF-YC structure gives insight into DNA binding and transcription regulation by CCAAT factor NF-Y. J Biol Chem. 2003;278:1336-45.

29. Rípodas C, Castaingts M, Clúa J, Blanco F, Zanetti ME. Annotation, phylogeny and expression analysis of the nuclear factor $Y$ families in common bean (Phaseolus vulgaris). Front Plant Sci. 2015;14:761.

30. Maity SN, de Crombrugghe B. Role of the CCAAT-binding protein CBF/NF-Y in transcription. Trends Biochem Sci. 1998;23:174-8.

31. Maity SN, de Crombrugghe B. Biochemical analysis of the B subunit of the heteromeric CCAAT-binding factor. A DNA-binding domain and a subunit interaction domain are specified by two separate segments. J Biol Chem. 1992;267:8286-92.

32. Xing Y, Fikes JD, Guarente L. Mutations in yeast HAP2/HAP3 define a hybrid CCAAT box binding domain. EMBO J. 1993;12:4647.

33. Hackenberg D, Wu Y, Voigt A, Adams R, Schramm P, Grimm B. Studies on differential nuclear translocation mechanism and assembly of the three subunits of the Arabidopsis thaliana transcription factor NF-Y. Mol Plant. 2012;5:876-88.
34. Shen B, Allen WB, Zheng P, Li C, Glassman K, Ranch J, et al. Expression of ZmLEC1 and ZmWRI1 increases seed oil production in maize. Plant Physiol. 2010;153:980-7.

35. Alam MM, Tanaka T, Nakamura H, Ichikawa H, Kobayashi K, Yaeno T, et al. Overexpression of a rice heme activator protein gene (OsHAP2E) confers resistance to pathogens, salinity and drought, and increases photosynthesis and tiller number. Plant Biotechnol J. 2014;13:85-96.

36. Liu GT, Wang JF, Cramer G, Dai ZW, Duan W, Xu HG, et al. Transcriptomic analysis of grape (Vitis vinifera L.) leaves during and after recovery from heat stress. BMC Plant Biol. 2012;12:174.

37. Fung RWM, Gonzalo M, Fekete C, Kovacs LG, He Y, Marsh E, et al. Powdery mildew induces defense-oriented reprogramming of the transcriptome in a susceptible but not in a resistant grapevine. Plant Physiol. 2008;146:236-49.

38. Wolters $H$, Jurgens $G$. Survival of the flexible: hormonal growth control and adaptation in plant development. Nat Rev Genet. 2009;10:305-17.

39. Grimplet J, Deluc LG, Tillett RL, Wheatley MD, Schlauch KA, Cramer GR, et al. Tissue-specific mRNA expression profiling in grape berry tissues. BMC Genomics. 2007:8:187.

40. Coombe BG, McCarthy MG. Dynamics of grape berry growth and physiology of ripening. Aust J Grape Wine Res. 2000;6:131-5.

41. Laporte P, Lepage A, Fournier J, Catrice O, Moreau S, Jardinaud MF, et al. The CCAAT box-binding transcription factor NF-YA1 controls rhizobial infection. J Exp Bot. 2013;65:481-94.

42. Soyano T, Kouchi H, Hirota A, Hayashi M. Nodule inception directly targets NF-Y subunit genes to regulate essential processes of root nodule development in Lotus japonicus. PLoS Genet. 2013;9, e1003352.

43. Li DY, Fu FY, Zhang HJ, Song FM. Genome-wide systematic characterization of the bZIP transcriptional factor family in tomato (Solanum lycopersicum L.). BMC Genomics. 2015:16:771.

44. Peng XJ, Liu H, Wang D, Shen SH. Genome-wide identification of the Jatropha curcas MYB family and functional analysis of the abiotic stress responsive gene JCMYB2. BMC Genomics. 2016;17:251.

45. Kumimoto RW, Zhang Y, Siefers N, Holt 3rd BF. NF-YC3, NF-YC4 and NF-YC9 are required for CONSTANS-mediated, photoperiod-dependent flowering in Arabidopsis thaliana. Plant J. 2010:63:379-91.

46. Cao S, Kumimoto RW, Siriwardana CL, Risinger JR, Holt 3rd BF. Identification and characterization of NF-Y transcription factor families in the monocot model plant Brachypodium distachyon. PLoS One. 2011;6, e21805.

47. Xu GX, Guo CC, Shan HY, Kong HZ. Divergence of duplicate genes in exonintron structure. Proc Natl Acad Sci U S A. 2012;109:1187-92.

48. Paradkar PN, Roth JA. Expression of the $1 \mathrm{~B}$ isoforms of divalent metal transporter (DMT1) is regulated by interaction of NF-Y with a CCAAT-box element near the transcription start site. J Cell Physiol. 2007;211:183-8.

49. Ni Z, Hu Z, Jiang Q, Zhang H. GmNFYA3, a target gene of miR169, is a positive regulator of plant tolerance to drought stress. Plant Mol Biol. 2013;82:113-29.

50. Tan H, Yang X, Zhang X, Qu C, Mu J, Fu F, et al. Enhanced seed oil production in canola by conditional expression of Brassica napus LEAFY COTYLEDON1 and LEC1-LIKE in developing seeds. Plant Physiol. 2011:156:1577-88.

51. Mendes A, Kelly AA, van Erp H, Shaw E, Powers SJ, Kurup S, et al. bZIP67 regulates the omega-3 fatty acid content of Arabidopsis seed oil by activating fatty acid desaturase3. Plant Cell. 2013;25:3104-16.

52. Kwong RW, Bui AQ, Lee H, Kwong LW, Fischer RL, Goldberg RB, et al. LEAFY COTYLEDON1-LIKE defines a class of regulators essential for embryo development. Plant Cell. 2003:15:5-18.

53. $\mathrm{Mu} \mathrm{J}, \mathrm{Tan} \mathrm{H}$, Hong $\mathrm{S}$, Liang $\mathrm{Y}$, Zuo J. Arabidopsis transcription factor genes NF-YA1, 5, 6, and 9 play redundant roles in male gametogenesis, embryogenesis, and seed development. Mol Plant. 2013;6:188-201.

54. Fujita M, Fujita Y, Noutoshi Y, Takahashi F, Narusaka Y, Yamaguchi-Shinozaki K, et al. Crosstalk between abiotic and biotic stress responses: a current view from the points of convergence in the stress signaling networks. Curr Opin Plant Biol. 2006:9:436-42

55. de Silva JM, Arrabaca MC. Contributions of soluble carbohydrates to the osmotic adjustment in the C 4 grass Setaria sphacelata: a comparison between rapidly and slowly imposed water stress. J Plant Physiol. 2004;161:551-5.

56. Garg AK, Kim JK, Owens TG, Ranwala AP, Choi YD, Kochian LV, et al. Trehalose accumulation in rice plants confers high tolerance levels to different abiotic stresses. Proc Natl Acad Sci U S A. 2002:99:15898-903.

57. Jin J, Zhang H, Kong L, Gao G, Luo J. PlantTFDB 3.0: a portal for the functional and evolutionary study of plant transcription factors. Nucleic Acids Res. 2014;42:D1182-7. 
58. Larkin MA, Blackshields G, Brown NP, Chenna R, Mcgettigan PA, Mcwilliam H, et al. Clustal W and Clustal X version 2.0. Bioinformatics. 2007;23:2947-8.

59. Tamura K, Peterson D, Peterson N, Stecher G, Nei M, Kumar S. MEGA5: Molecular Evolutionary Genetics Analysis using Maximum Likelihood, Evolutionary Distance, and Maximum Parsimony Methods. Mol Biol Evol. 2011;28:2731-9.

60. Reid KE, Olsson N, Schlosser J, Peng F, Lund ST. An optimized grapevine RNA isolation procedure and statistical determination of reference genes for real-time RT-PCR during berry development. BMC Plant Biol. 2006;6:27.

61. Livak K, Schmittgen TD. Analysis of relative gene expression data using real-time quantitative PCR and the 2(-Delta Delta C(T)) Method. Methods. 2001;25:402-8.

62. Wong DCJ, Sweetman C, Drew DP, Ford CM. VTCdb: a gene co-expression database for the crop species Vitis vinifera (grapevine). BMC Genomics. 2013;14:882.

63. Anna M, Maryse L, Rossitza A. Profiling of sugar transporter genes in grapevine coping with water deficit. FEBS Lett. 2014;588:3989-97.

64. Peng WT, Lee YW, Nester EW. The phenolic recognition profiles of the Agrobacterium tumefaciens VirA protein are broadened by a high level of the sugar binding protein ChvE. J Bacteriol. 1998;180:5632-8.

\section{Submit your next manuscript to BioMed Central} and we will help you at every step:

- We accept pre-submission inquiries

- Our selector tool helps you to find the most relevant journal

- We provide round the clock customer support

- Convenient online submission

- Thorough peer review

- Inclusion in PubMed and all major indexing services

- Maximum visibility for your research

Submit your manuscript at www.biomedcentral.com/submit 